\title{
The role of simulators, models, phantoms. Where's the evidence?
}

K. Matsuda', R. H. Hawes², A. V. Sahai ${ }^{3}$, H. Tajiri ${ }^{4}$

${ }^{1}$ Department of Endoscopy, The Jikei University Aoto Hospital, Tokyo, Japan

2 Digestive Disease Center, Medical University of South Carolina, Charleston, South Carolina, USA

${ }^{3}$ Department of Gastroenterology, University of Montreal, Quebec, Canada

${ }^{4}$ Department of Gastroenterology \& Hepatology, The Jikei University School of Medicine, Tokyo, Japan

Introduction

Training to a level of competency for EUS is challenging and involves a long learning curve. Exposure to EUS cases may be limited and thus learning tools need to be developed to decrease the number of supervised examinations in patients required to master this. Also, the trainees have to reach a certain skill level in the limited period of EUS fellowship. A few learning tools have been developed to allow a faster learning of EUS and EUS-FNA.

Phantom

Olympus (Olympus, Inc., Tokyo, Japan) has developed an EUSFNA phantom, which consists of a box ( $50 \mathrm{~cm}$ in length) with a

Correspondence: Koji Matsuda, MD, PhD · Phone: +81-3-3603-2111, ex. 3181 . Fax: +81-3-34594524

Bibliography: Endoscopy 2006; 38 (S1): S61-S64 @ Georg Thieme Verlag KG Stuttgart · New York · ISSN 0013-726X · DOI 10.1055/s-2006-946656 
hole in the center to simulate the esophagus and different types and sizes of silicon block to simulate lymph nodes or cystic lesions. (Fig. 1) The advantage of this model is that it is simple, easy to use and conveniently stored and transported. Sorbi et al. reported another type of phantom using commonly available materials (barium enema bag, plastic tube, standard agar, etc.). [1] Both phantoms can aid in learning the basics of EUS-FNA especially needle manipulation and positioning of the echoendoscope relative to the target lesion. However, phantoms cannot simulate or teach other EUS-related techniques, and are inadequate "real-life" simulators.

\section{Computer-based simulator}

EUS Mentor (Simbionix, Tel Hashomer, Israel) represents an addition to a computer-based endoscopic simulator platform and was developed for radial and linear-array EUS [2] (Fig. 2). It provides real-time computer simulated EUS images based on human anatomy. As of now, this system cannot create natural resistance for the tools through the working channels and thus

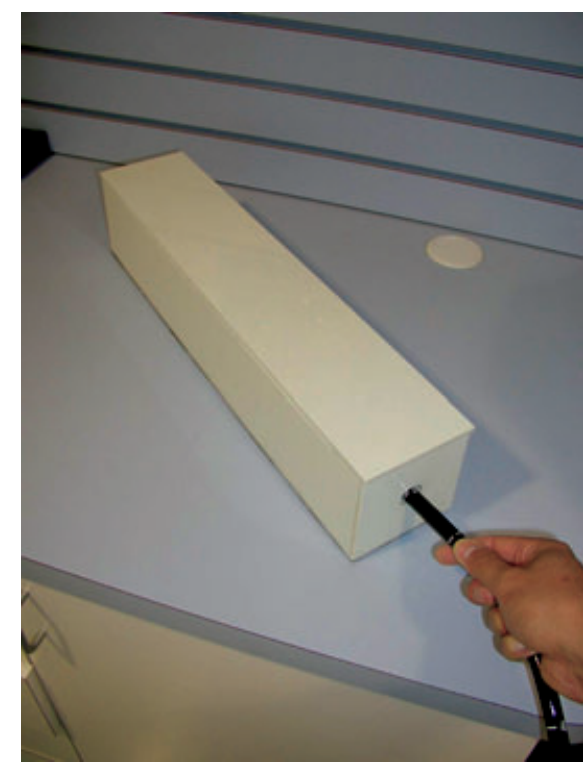

Fig. 1 Outlook of EUS-FNA box.

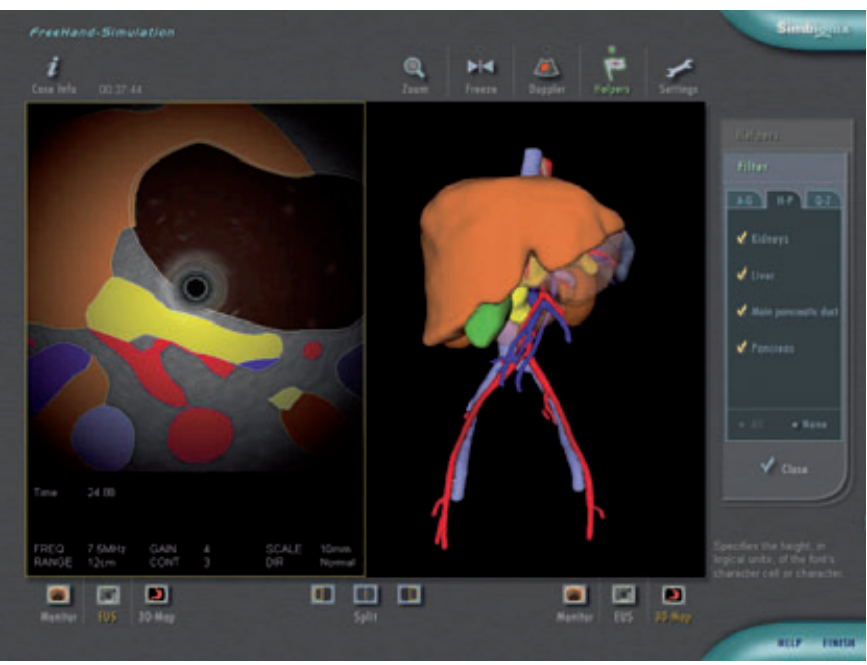

Fig. 2 Radial EUS images and anatomical view on EUS Mentor.

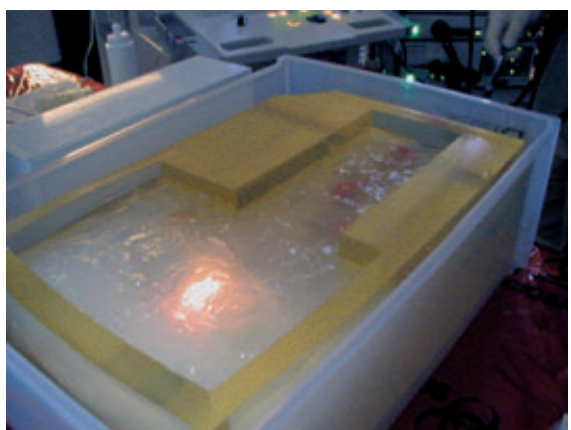

Fig. 3 Outlook of EUS RK model. cannot simulate EUS-FNA. Burmester et al. reported another type of simulator, which was especially designed for linear-array EUS with the three dimensional integration of the real anatomy of the cadavers [3]. This interactive simulation system is very useful to learn the basic anatomic structure using the linear array echoendoscope. However, there is no function for scope or needle manipulation.

\section{EUS RK model}

The Erlanger model is now widely used for training in therapeutic EGD and colonoscopy as well as ERCP [4]. This model was modified to accommodate EUS training [5,6] (Fig. 3). It consists of special made silicon case housing the ex-vivo pig stomach surrounded by a gelatin medium to provide acoustic coupling. Grapes were embedded in gelatin simulate lymph nodes. The whole preparation is labor intensive and takes about 6 hours excluding preparation of pig organs, and can last 2 to 3 days in the refrigerator after use. This model can generate real-time EUS images of tissue and is the most realistic simulator of EUS-FNA besides the live pig model. This model has a potential to contribute to the development of therapeutic EUS tools in the future. This model was used in the learning center of 13th and 14th International Symposium on EUS as well as hands-on training courses in Japan, Singapore, USA, and Europe. This model would be suitable for relatively large training centers but needs to be simplified and made less labor intensive.

\section{Live pig}

The swine has been the most popular model for teaching EUS. The anatomy is similar in many respects to the human, especially the pancreas, left kidney and celiac axis. The live pig is realistic, however, it may be difficult to acquire, is expensive and ethical issues often limit its availability. Bhutani et al. reported on the efficacy of a swine model for teaching EUS tutorials [7] as well as for teaching interventional EUS by making a "pseudo-lesion" with the injection of saline [8]. The conclusion was that this model was helpful for understanding manipulation of the scope and needle manipulation. The live pig can be an excellent option for teaching EUS but requires an animal care facility.

\section{How can we measure the efficacy of learning tools for EUS?}

For colonoscopy, Ahlberg et al. reported that endoscopic simulators actually improved the early part of the learning curve in co- 
lonoscopy training [9]. In the field of ERCP, Sedlack et al. reported the direct comparison between the live pig, a computer simulator and the EASIE model for ERCP [10]. The EASIE model scored the highest on indices of realism, usefulness, and performance, although this reached statistical significance only for "ease of use". The computer simulator scored significantly lower in most realism scores, although it was felt to be the one model most easily incorporated into a training system.

In the case of EUS, there is no direct comparison demonstrating the actual efficacy of each type of learning tool for EUS and EUSFNA, although a few reports suggested that they represented an overall aid in education $[3,5-7]$. We conducted a survey to assess the impact of the phantom, a computer simulator, the modified Erlanger (RK) model and the live pig as a learning tool. Eight EUS experts (Peter Vilmann, Mitsuhiro Kida, Kenneth Binmoeller, Vipulroy Rathod, Anand Sahai, Greg Zuccaro, Robert Hawes, Koji Matsuda) were queried regarding the teaching utility of these various tools. If they had no experience in a particular tool, no score was counted.

\section{Experience of each tool for educational purpose}

The experience of each tool by eight EUS experts is shown in Table 1. Only the ex-vivo model (RK model) had been used by all experts. The fewest number of experts had experience with the computer simulator.

Table 1 Experience of each tool as an educational tools for EUS and EUS-FNA

\begin{tabular}{llll}
\hline EUS-FNA box & EUS Mentor & EUS RK model & Live pig \\
\hline $7 / 8$ & $5 / 8$ & $8 / 8$ & $6 / 8$ \\
\hline
\end{tabular}

\section{Scores for realism compared with a human EUS}

The realism was evaluated for each system. The following criteria were scored on a 7-point Likert scale ( 1 = very unrealistic, 7 = very realistic): (1) anatomy of mediastinum, (2) anatomy of pancreatic body and celiac axis (3) visualization, (4) scope manipulation, (5) needle manipulation (Table 2 ). In terms of anatomy, live pig scored best, followed by EUS Mentor and EUS RK model. Regarding scope manipulation, the live pig and EUS Mentor scored best, followed by EUS RK model. For needle manipulation, the live pig was best, followed by the ex-vivo system and EUS-FNA box.

Scores for utility as an educational tool for EUS and EUS-FNA

A similar scale was used to assess the utility of each tool for teaching of EUS and EUS-FNA (1 = not useful at all, 7 = very useful) (Table 3). In terms of overall utility for teaching EUS, the EUS Mentor scored slightly higher than the live pig, followed by EUS RK model. In terms of usefulness in teaching EUS-FNA, the live pig scored highest, followed by RUS RK model and EUS-FNA box.

\section{Ordered rankings for four learning tools (Table 4)}

Eight EUS experts directly compared the four tools by ranking them in order for the following: (1) overall simulation realism ( 1 = most, 4 = least), (2) utility as an educational modality ( 1 = most, 4 = least), ( 3 ) ease of use ( 1 = easiest, 4 = most difficult), (4) ease of incorporation into a fellowship training program ( 1 = easiest, 4 = most difficult). If the experts did not have the experience using one tool, no rank was counted.

The EUS-FNA box was rated highest in "ease of use" and "ease of incorporating into fellowship training", whereas it was lowest in "overall simulation realism" and "scores for utility as an educa-

Table 2 Scores for realism compared with a human EUS

\begin{tabular}{lllll}
\hline & EUS-FNA box & EUS Mentor & EUS RK model & Live pig \\
\hline Anatomy of mediastinum & $1(1-3)$ & $\mathbf{4 . 5}(1-6)$ & $3(1-6)$ & $4(1-6)$ \\
\hline Anatomy of pancreatic body and celiac axis & $1(1-3)$ & $4(4-6)$ & $4(1-5)$ & $\mathbf{5 ( 1 - 6 )}$ \\
\hline Visualization & $4(2-6)$ & $5(4-6)$ & $5.5(3-6)$ & $\mathbf{6 ( 4 - 7 )}$ \\
\hline Scope manipulation & $3(2-5)$ & $\mathbf{6 ( 4 - 6 )}$ & $4.5(3-7)$ & $\mathbf{6 ( 4 - 7 )}$ \\
\hline Needle manipulation & $5(3-6)$ & $4(1-6)$ & $5(4-7)$ & $\mathbf{6 ( 5 - 7 )}$ \\
\hline
\end{tabular}

Median scores (range) are based on a 7-point Likert scale ( 1 = very unrealistic, 7 = very realistic). Scores in bold represent the highest score in each category.

Table 3 Scores for utility as an educational tool for EUS and EUS-FNA

\begin{tabular}{lllll}
\hline & EUS-FNA box & EUS Mentor & EUS RK model & Live pig \\
\hline Usefulness in teaching EUS & $3(1-6)$ & $\mathbf{6}(4-6)$ & $4(2-7)$ & $5.5(4-6)$ \\
Usefulness in teaching EUS-FNA & $5(4-6)$ & $3(1-6)$ & $5(4-7)$ & $\mathbf{6 ( 5 - 7 )}$ \\
\hline
\end{tabular}

Median scores (range) are based on a 7-point Likert scale ( 1 = not useful at all, 7 = very useful). Scores in bold represent the highest score in each category. 


\begin{tabular}{lllll}
\hline Overall simulation realism & $3(3-4)$ & $2(1-3)$ & $2(1-3)$ & $\mathbf{1}(1-2)$ \\
\hline Scores for utility as an educational tool & $3(3-4)$ & $2(1-3)$ & $2(1-3)$ & $\mathbf{1}(1-2)$ \\
\hline Ease of use & $\mathbf{1}(1-3)$ & $2(1-2)$ & $2.5(1-4)$ & $3(2-4)$ \\
\hline Ease of incorporating into fellowship training & $\mathbf{1}(1-3)$ & $2(1-3)$ & $2(1-4)$ & $3(3-4)$ \\
\hline
\end{tabular}

Median ranking (range) are based on a 4-point hierarchy (1 = best, 4 = least). Scores in bold represent the best median ranking in each category.

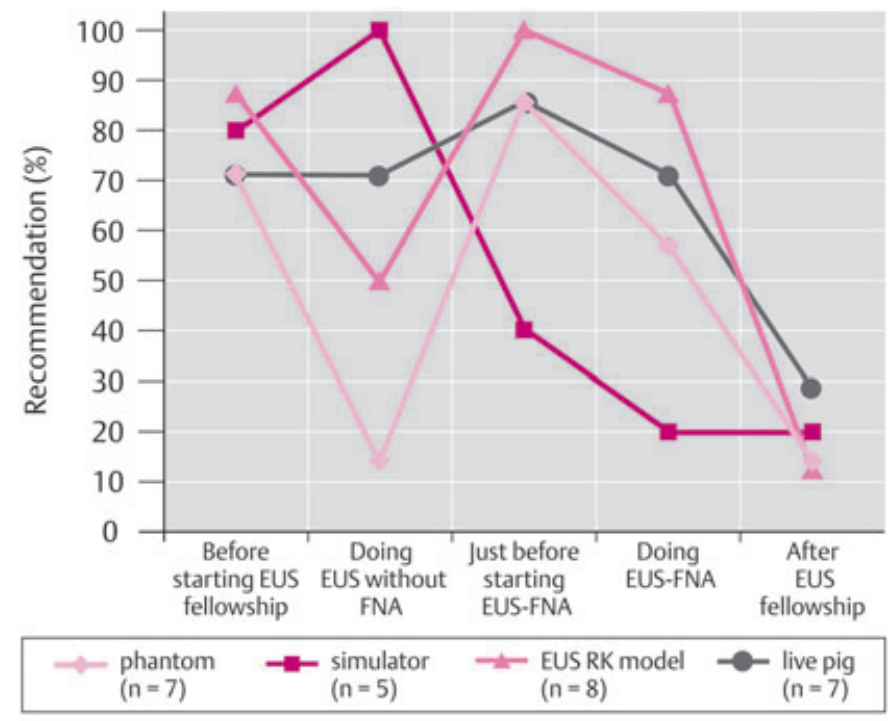

Fig. 4 Recommendation of learning tools in each teaching session by EUS experts.

tional tool". The live pig ranked highest in "overall simulation realism" and "scores for utility as an educational tool". However, the live pig was inferior to the other tools for "ease of use" and "ease of incorporating into fellowship training".

Recommendation of learning tools in each teaching session by EUS experts

The eight EUS experts marked the utility of each of the learning tools relative to what stage the trainees were along the learning curve (Fig. 4). EUS Mentor was recommended highest when "doing EUS without FNA", followed by "before starting EUS fellowship", whereas the EUS RK model and phantom was recommended most in "just before starting EUS-FNA". The live pig was recommended throughout the training process. This type of study will be necessary to show the evidence of the efficacy of learning tools from the point of trainee.

\section{Summary}

There are a number of options to assist the trainee to achieve competency in EUS and EUS-FNA. Although no randomized controlled trial with these tools have been performed to definitely determine their role in training, this survey of EUS experts sug- gest they can have a positive role. The authors believe that it is critically important to develop practical and useful leaning tools for EUS and EUS-FNA and that these tools will significantly aid in the teaching of EUS. Further study from the point of trainees would be necessary.

\section{References}

${ }^{1}$ Sorbi D, Vazquez-Sequeiros E, Wiersema MJ. Simple phantom for learning EUS-guided FNA. Gastrointest Endosc 2003; 57 (4): 580 - 583

2 Bar-Meir S. A New Endoscopic Simulator. Endoscopy 2000; 32 (11): $898-900$

${ }^{3}$ Burmester E, Leineweber T, Hacker S et al. EUS Meets Voxel-Man: three-dimensional anatomic animation of linear-array endoscopic ultrasound images. Endoscopy 2004; 36 (8): 26-30

${ }^{4}$ Neumann M, Mayer G, Ell C et al. The Erlangen Endo-Trainer: Life-Like Simulation for Diagnostic and Interventional Endoscopic Retrograde. Cholangiography Endoscopy 2000; 32 (11): 906 - 910

${ }^{5}$ Matsuda K, Hawes R. How shall we experience EUS before the actual first procedure? - Development of modified Erlangen Active Simulator for Interventional Endoscopy (EASIE) model for EUS training. Gastrointest Endosc 2002; 56 (4): S143

${ }^{6}$ Matsuda K, Tajiri H, Hawes RH. How shall we experience EUS and EUSFNA before the first procedure? - the development of learning tools. Digestive Endoscopy 2004; 16: S236-239

${ }^{7}$ Bhutani MS, Hoffman BJ, Hawes RH. A swine model for teaching endoscopic ultrasound (EUS) imaging and intervention under EUS guidance. Endoscopy 1998; 30 (7): 605-609

${ }^{8}$ Bhutani MS, Aveyard M, Stills HF Jr. Improved model for teaching interventional EUS. 2000; 52 (3): 400-403

${ }^{9}$ Ahlberg G, Hultcrantz R, Jaramillo E et al. Virtual reality colonoscopy simulation: a compulsory practice for the future colonoscopist? 2005; 37 (12): $1198-1204$

${ }^{10}$ Sedlack R, Petersen B, Binmoeller K et al. A direct comparison of ERCP teaching models. Gastrointest Endosc 2003; 57: 886-890 